\title{
PURIFICATION AND SOME PROPERTIES OF RECOMBINANT SHEEP ANGIOTENSINOGEN EXPRESSED IN CHINESE HAMSTER OVARY CELLS
}

\author{
Masahiro Nagase ${ }^{1,4}$, Fumiaki Suzuki ${ }^{2,3}$, Yoshinori SaWai ${ }^{1}$, Takenori Orihashi ${ }^{1}$, Yoshito Inui ${ }^{1}$, \\ TSutomu Nakagawa ${ }^{1}$ and Yukio Nakamura ${ }^{1}$ \\ 'Department of Biotechnology, Faculty of Agriculture and ${ }^{2}$ Molecular Genetics Research Center, Gifu University, Gifu 501-11, \\ Japan
}

\begin{abstract}
We established an expression system for large amount of recombinant sheep angiotensinogen (sAngn) in Chinese hamster ovary cells. The recombinant sAngn (21.6 mg) was purified by a single column chromatography to a homogeneous level on sodium dodecyl sulphate-polyacrylamide gel electrophoresis from 2.71 of conditioned medium. Human renin cleaved only a Leu ${ }^{10}-\mathrm{Leu}^{11}$ bond in sAngn, although $\mathrm{Leu}^{11}-\mathrm{Val}^{12}$ was another candidate for scissile peptide bond. The $K_{\mathrm{m}}$ and $k_{\text {cat }}$ values for the reaction of human renin with sAngn were $0.21 \mu \mathrm{M}$ and $150 \mathrm{~min}^{-1}$, respectively. Recombinant rat renin could also react with the sAngn. The $K_{\mathrm{m}}$ and $k_{\text {cat }}$ for the reaction were $0.84 \mu \mathrm{g}$ and $78 \mathrm{~min}^{-1}$, respectively. Purified sAngn was stable at temperature up to $50^{\circ} \mathrm{C}$, but rapidly inactivated at that higher than $55^{\circ} \mathrm{C}$.
\end{abstract}

Angiotensinogen (Angn) is a prohormone that is synthesized in the liver and is cleaved by renin (EC 3.4.23.15) to release angiotensin I (Ang I) (17). The decapeptide is processed by angiotensin-converting enzyme (EC 3.4.15.1) to an active octapeptide hormone, angiotensin II, which controls blood pressure and electrolyte balance.

Renin belongs to aspartic protease family $(7,10)$. Most of aspartic proteases react with their substrates at acidic $\mathrm{pH}$. On the other hand, renin acts with Angn at neutral $\mathrm{pH}$ in the blood

Abbreviations: sAngn, sheep angiotensinogen; Angn, angiotensinogen; Ang I, angiotensin I; SDS-PAGE, sodium dodecyl sulphate-polyacrylamide gel electrophoresis; $\mathrm{CHO}$, Chinese hamster ovary; dhfr, dihydrofolate reductase; FBS, fetal bovine serum; MTX, methotrexate

${ }^{3}$ Correspondence to: F. Suzuki at the above address. Tel: 058-293-3174; Fax:058-293-3172; E-mail: aob3073@ cc.gifu-u.ac.jp

${ }^{4}$ Present address: Department of Bioresource Science, Faculty of Agriculture, Kagawa University, Miki, Kagawa 761-07, Japan circulation. The reaction mechanism of renin is almost unclear, although kinetic studies of renin with synthesized peptide substrates have been reported $(2,5,14)$. Kinetic parameters of human renin with peptide substrates were different from those with plasma Angn $(2,6)$. In order to elucidate the mechanism of renin-angiotensinogen reaction, a sufficient amount of Angn is required. Plasma sheep angiotensinogen (sAngn) is well-known to be a better substrate for human renin, and has therefore been used in clinical study on renin. In this study, we established a Chinese hamster ovary (CHO) cell line that produced a large amount of recombinant sAngn, and determined some of its properties.

\section{MATERIALS AND METHODS \\ Construction of an Expression Vector of sAngn}

A plasmid pBSsAG was digested with EcoR I, treated with DNA polymerase I (Klenow fragment), and followed by ligation with BamH I linker. The modified plasmid with $B a m \mathrm{H}$ I site at the 


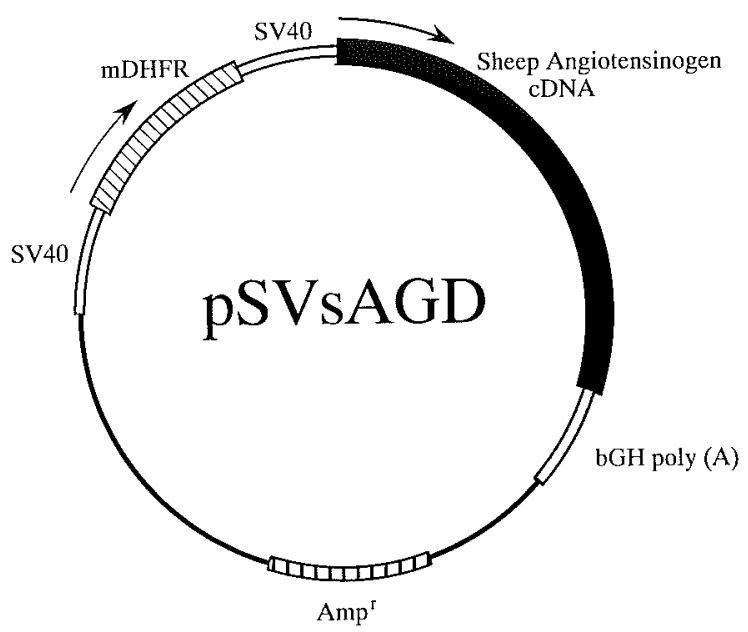

Fig. 1 Structure of an expression vector, pSVsAGD. SV40, early promoter and replication origin of SV40; mDHFR, mouse dihydroforate reductase gene; bGH poly(A), polyadenylation signal from bovine growth hormone gene; $\mathrm{Amp}^{r}$, ampicilin resistant gene

$5^{\prime}$-end of sAngn cDNA was further digested with $X b a \mathrm{I}$ and processed again to obtain sAngn cDNA with $B g l$ II site at the $3^{\prime}$-end. The entire sequence of sAngn cDNA with 1,938 bp was ligated into $B a m \mathrm{H}$ I site of pSVD vector (18) to obtain an expression vector for $\mathrm{CHO}$ cells, pSVsAGD (Fig. $1)$.

\section{Establishment of CHO Cell Line Producing sAngn}

The vector, pSVsAGD, was transfected into dihydrofolate reductase (dhfr)-deficient $\mathrm{CHO}$ cells (DXB-11) by a calcium phosphate-mediated method using CellPhect Transfection kit (Pharmacia, Uppsala, Sweden). The cells harboring the vector with (dhfr) gene were isolated by culturing in the selection medium, Dulbecco's modified Eagle medium supplemented with $5 \%$ dialyzed fetal bovine serum, 100 units $/ \mathrm{ml}$ penicillin, 100 $\mu \mathrm{g} / \mathrm{ml}$ streptomycin, $2 \mathrm{mM}$ L-glutamin and $100 \mu \mathrm{M}$ non essential amino acid. Production of recombinant sAngn was enhanced by methotrexate (MTX, Sigma, St. Louis, U.S.A.). The dose amount was stepwise increased from 0 to $0.5 \mu \mathrm{M}$ as shown in Fig. 2. It took for 10 months for the overall procedure of the enhancement. The amount of sAngn secreted into the medium was determined as described previously (13). Briefly, sAngn preparation was incubated with excess amount of recombi-

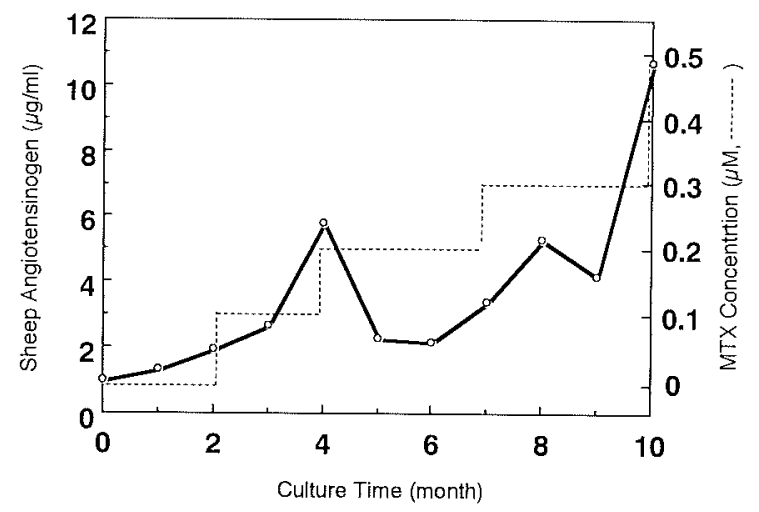

Fig. 2 Stepwise enhancement of sAngn productivity in $\mathrm{CHO}$ cells by MTX. Concentration of recombinant sAngn secreted into the medium was monitored by standard assay method at each step.

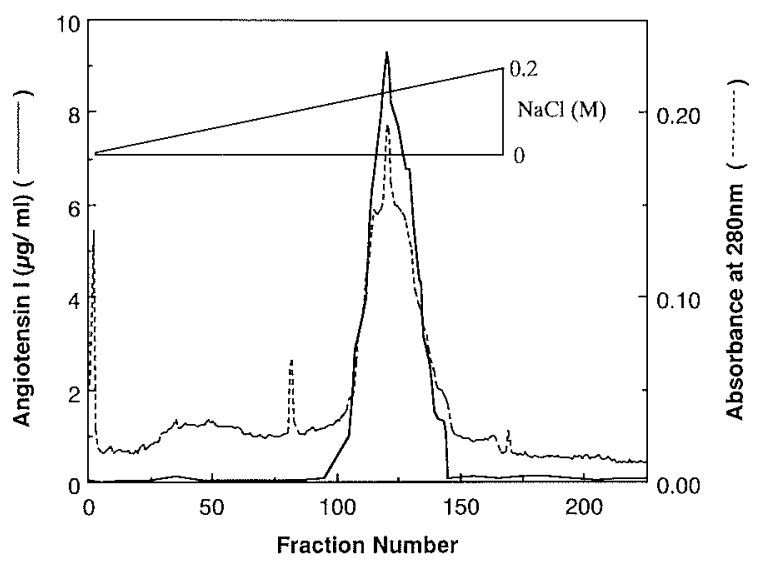

Fig. 3 A cation exchange chromatography of sAngn on a CM-Toyopearl 650 column. Recombinant sAngn fraction, obtained concentration and dialysis of 2.71 of conditioned medium, was applied into a CM-Toyopearl 650 $\mathrm{M}$ column (Tosoh, Tokyo; $2 \times 20 \mathrm{~cm}$ ) preequilibrated with $10 \mathrm{mM}$ sodium acetate buffer (pH 5.0). Recombinant sAngn was eluted by a $\mathrm{NaCl}$ gradient from 0 to 0.3 $\mathrm{M}$ at a flow rate of $0.625 \mathrm{ml} / \mathrm{min}$ at $4^{\circ} \mathrm{C}$.

nant human renin in the presence of $10 \mathrm{mM}$ diisopropyl fluorophospate, $10 \mathrm{mM}$ EDTA at $\mathrm{pH} 6.5$ and $37^{\circ} \mathrm{C}$ for $0.5,1$ and $2 \mathrm{~h}$, and Ang I released from sAngn was assayed by Ang I-ELISA $(11,21)$. The plateau level of Ang I produced was, in this study, represented as the amount of sAngn. Protein concentration was measured using a Protein Assay kit (BIO-RAD, Hercules, U.S.A.). The productivity of sAngn in the cells was increased 10-fold by this MTX enhancement procedure (Fig. 2). The CHO 
Table 1 Purification of Sheep Angiotensinogen

\begin{tabular}{lccccc}
\hline Purification step & $\begin{array}{c}\text { Total protein } \\
(\mathrm{mg})\end{array}$ & $\begin{array}{c}\text { Total Ang I } \\
(\mu \mathrm{g})\end{array}$ & $\begin{array}{c}\text { Specific } \\
\text { amount } \\
(\mu \mathrm{g} \mathrm{Ang} \mathrm{I/mg})\end{array}$ & $\begin{array}{c}\text { Purification } \\
(\text { fold })\end{array}$ & $\begin{array}{c}\text { Recovery } \\
(\%)\end{array}$ \\
\hline $\begin{array}{l}\text { Conditioned } \\
\text { medium }\end{array}$ & 155 & 838 & 5.41 & 1 & 100 \\
Concentration & 137 & 836 & 6.01 & 1.11 & 99.9 \\
Dialysis & 74.6 & 694 & 9.30 & 1.72 & 82.8 \\
CM-Toyopearl & 21.6 & 455 & 21.1 & 3.90 & 54.3 \\
\hline
\end{tabular}

cell line obtained in this protocol was further cultured under the same conditions as the final enhancement, and was used in this study. The sAngn productivity has almost unchanged for more than 2 years.

\section{Recombinant Human and Rat Renins}

Human renin and rat renin used in this study were purified by the methods previously described (18, 23). Both $\mathrm{CHO}$ cell lines that express human renin or rat renin were generously gifted from Professor K. Murakami of the Tsukuba University.

\section{RESULTS AND DISCUSSION}

\section{Purification of Recombinant sAngn}

The 2.71 of conditioned medium was concentrated by polyethylene glycol \#20000 and dialyzed against a sufficient amount of $10 \mathrm{mM}$ sodium acetate buffer ( $\mathrm{pH}$ 5.0). The dialyzate was applied to a CM-Toyopearl $650 \mathrm{M}$ column preequilibrated with $10 \mathrm{mM}$ sodium acetate buffer (pH 5.0). Recombinant sAngn was eluted by a $\mathrm{NaCl}$ gradient from 0 to $0.3 \mathrm{M}$, as shown in Fig. 3. The recombinant sAngn was purified by 3.90 -fold with an overall yield of $54.3 \%$ by the single step purification as summarized in Table 1.

\section{Molecular Mass of Recombinant sAngn}

Purified sAngn was electrophoresed on sodium dodecyl sulphate-polyacrylamide gel electrophoresis (SDS-PAGE) according to the method of Laemmli (9), with additional sodium thioglycollate $(100 \mathrm{mM})$ in cathodic buffer. As shown in Fig. 4, the migration distance of recombinant sAngn was identical to that of native sAngn $(5,14)$ and the molecular mass was estimated to be $56 \mathrm{kDa}$ (Fig. 4).

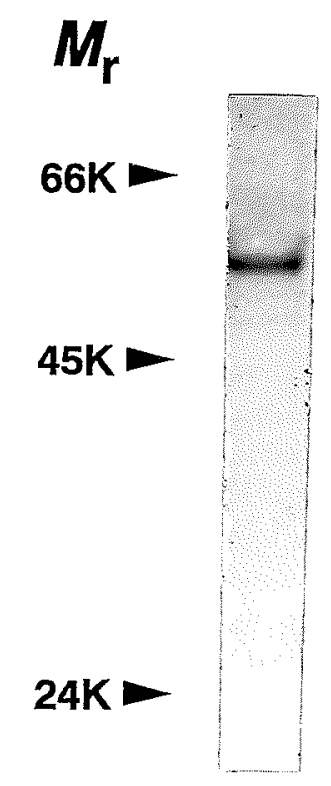

Fig. 4 SDS-PAGE of purified recombinant sAngn. Purified sAngn $(5 \mu \mathrm{g})$ was electrophoresed on $7.5 \%$ SDS-PAGE and stained by Coomassie Blue R-250.

\section{N-Terminal Amino Acid Sequence of Recombinant sAngn}

The purified sAngn, separated by SDS-PAGE under reduced conditions, was transferred onto polyvinylidene difluoride membrane and stained by Coomassie Blue R-250. The sAngn was subjected to automated protein sequencer (model Procise 491, PE-ABI). The N-terminal amino acid sequence was determined to be Asp-Arg-Val-TyrIle-His-Pro-Phe-His-Leu-Leu-Val-His-Ser-Lys-SerAsn-(Xaa)-Asp-Gln-Leu-Glu-Lys-Pro-Ser-Val-Glu. This result was identical to that of native sAngn previously reported (4). These results indicated that recombinant sAngn was normally processed in the $\mathrm{CHO}$ cells and secreted into the culture medium. 
Table 2 Kinetic Parameters of sAngn with Renins of Human and Rat at pH 6.5

\begin{tabular}{lccc}
\hline Renin species & $\begin{array}{c}K_{\mathrm{m}} \\
(\mu \mathrm{M})\end{array}$ & $\begin{array}{c}k_{\text {cat }} \\
\left(\mathrm{min}^{-1}\right)\end{array}$ & $\begin{array}{c}k_{\text {cat }} / K_{\mathrm{m}} \\
\left(\mu \mathrm{M}^{-1} \mathrm{~min}^{-1}\right)\end{array}$ \\
\hline Human & 0.21 & 150 & 721 \\
Rat & 0.84 & 78 & 93 \\
\hline
\end{tabular}

\section{The Cleavage Site of Human Renin in sAngn}

Renins of human and rat cleave the peptide bond of Leu ${ }^{10}-\mathrm{Val}^{11}$ and that of $\mathrm{Leu}^{10}-\mathrm{Leu}^{11}$ in Angns of human and rat, respectively. Additionally, amino acid sequences near the scissile peptide bond of human and rat Angns are His ${ }^{9}-\mathrm{Leu}-\mathrm{Val}-\mathrm{Ile}^{12}$ (8, 19, 22) and His ${ }^{9}-L e u-L e u-T_{y}{ }^{12}(1,15)$, respectively. The amino acid sequence from position 9 th to 12 th in sAngn is His-Leu-Leu-Val $(4,12)$. Two candidate sites for cleavage by human renin would therefore be Leu ${ }^{10}-\mathrm{Leu}^{11}$ and $\mathrm{Leu}^{11}-\mathrm{Val}^{12}$ in sAngn. The N-terminal amino acid sequence of the sAngn after incubated with a sufficient amount of human renin was determined to be Leu-Val-His-Ser-LysSer-Asn-Xaa-Asp-Gln-Glu-Lys-Pro-Ser-Val-Glu. The amino acid sequence of this modified sAngn started from the position of $\mathrm{Leu}^{11}$ in the sAngn. Human renin was determined to cleave sAngn at the $\mathrm{Leu}^{10}{ }^{10} \mathrm{Leu}^{11}$ bond to produce Ang I peptide and des(Ang I) Angn. These results indicate that renin reaction prefers the peptide bond of 10th and 11th residue to side chain specificity under these conditions.

\section{Kinetic Parameters of Human or Rat Renin with Recombinant sAngn}

The $K_{\mathrm{m}}$ and $k_{\text {cat }}$ of human renin with sAngn were $0.21 \mu \mathrm{M}$ and $150 \mathrm{~min}^{-1}$, respectively (Table 2). This is similar to that for plasma sAngn reported previously $(3,20)$. On the other hand, The $K_{\mathrm{m}}$ and $k_{\text {cat }}$ of rat renin with sAngn were determined to be $0.84 \mu \mathrm{M}$ and $78 \mathrm{~min}^{-1}$, respectively (Table 2 ), indicating that recombinant sAngn can also be used for the study of rat renin.

\section{Thermostability of Recombinant sAngn}

Thermostability of sAngn was determined by measuring the residual reactivity of Angn with human renin after incubation at various temperatures for 30 min. As shown in Fig. 5, sAngn was stable at temperature up to $50^{\circ} \mathrm{C}$ and completely inactivated

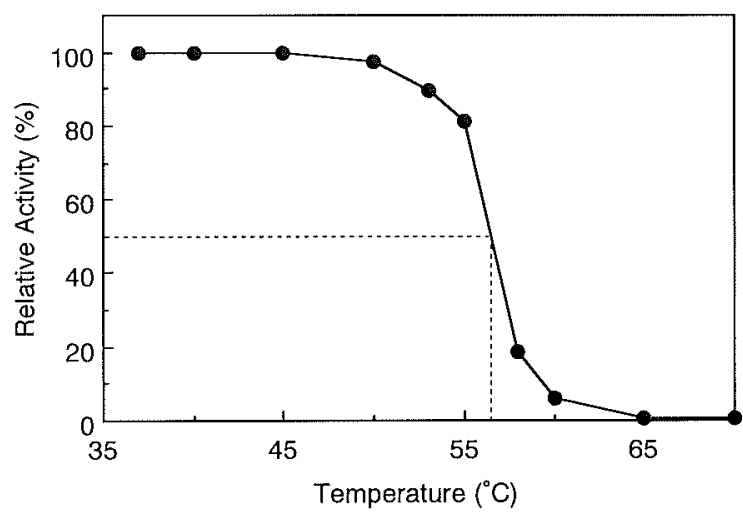

Fig. 5 Thermostability of recombinant sAngn. The reaction mixture, contained $57 \mu \mathrm{g} / \mathrm{ml}$ recombinant sheep angiotensinogen, $60 \mathrm{mM}$ sodium phosphate (pH 6.0), 10 $\mathrm{mM}$ diisopropyl fluorophosphate and $10 \mathrm{mM}$ ethylenediamine tetraacetate, was incubated at indicated temperature for $30 \mathrm{~min}$, and then cooled on ice to proceed the reaction with human renin.

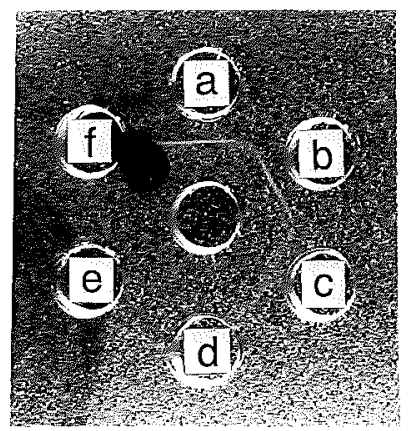

Fig. 6 Double immunodiffusion analysis of anti-sAngn antiserum against various source of Angns. Native angiotesinogens $(2-3 \mu \mathrm{g})$ of sheep (well b), human (well c), rat (well d) and porcine (well e) were partially purified from their plasma as previously described (11). Blank well without any serum was set at well $\mathrm{f}$. AntisAngn antiserum (1:1,000 dilution) and recombinant sAngn were applied in the center well and well a, respectively.

at $65^{\circ} \mathrm{C}$ under these conditions.

\section{Production of Anti-sAngn Antibodies and Their Immunochemical Property}

Purified recombinant sAngn was emulsified with complete Freund's adjuvant and 100-200 $\mu$ g quantities of the antigen were intradermally injected into multiple sites of New Zealand White rabbits, followed by boosting at intervals of 1 week with 
the protein in incomplete Freund's adjuvant for five weeks. Eighteen weeks later, the same injection procedure was repeated. Anti-sAngn antiserum was obtained one week after the final booster. Ouchterlony double immunodiffusion analysis (16) showed that anti-sAngn antibodies did not bind with human, rat and porcine Angns, but did bind with recombinant and plasma sAngns (Fig. 6). These results show that sAngn antibodies obtained in this study have remarkably high specificity for species. These antibodies would be a useful tool for renin-Angn studies.

In this study, we have established a stable mammalian cell line producing a large amount of recombinant sAngn. This expression system will be useful to elucidate the mechanism of reninAngn reaction and to further investigate on the renin angiotensin system.

Received 3 September 1997; and accepted 3 October 1997

\section{REFERENCES}

1. Bouhnik J., Clauser E., Strosberg D., Menard J. and Corvol P. (1981) Rat angiotensinogen and des(angiotensin I) angiotensinogen: Purification, characterization, and partial sequencing. Biochemistry 20, 7010-7015

2. Cumin F., Le-Nguyen D., Castro B., Menard J. and Corvol P. (1987) Comparative enzymatic studies of human renin acting on pure natural or synthetic substrates. Biochim. Biophys. Acta 913, 10-19

3. Do Y. S., Shinagawa T., Tam H., Inagami T. and Hsueh W. A. (1987) Characterization of pure human renal renin. Evidence for a subunit structure. J. Biol. Chem. 262, 1037-1043

4. Fernley R. T., John M., Niall H. D. and Coghlan J. P. (1986) Purification and characterization of ovine angiotensinogen. Eur: J. Biochem. 154, 597-601

5. Green D. W., Aykent S., Grierse J. K. and Zupec M. E. (1990) Substrate specificity of recombinant human renal renin: Effect of histidine in the $\mathrm{P}_{2}$ subsite on $\mathrm{pH}$ dependence. Biochemistry 29, 3126-3133

6. Hatae T., Takimoto E., Fukamizu A., Hori H., Kimoto K. and Murakami K. (1992) Expression and purification of human angiotensinogen in Chinese hamster ovary cells. Biochim. Biophys. Acta 1121 (3), 335-338

7. Inagami T., Misono K. S. and Michelakis A. M. (1974) Definitive evidence for similarity in the acitve site of renin and acid protease. Biochem. Biophys. Res. Commun. 56, 503-509

8. Kageyama R., Ohkubo H. and Nakanishi S. (1984) Pri- mary structure of human preangiotensinogen deduced from the cloned cDNA. Biochemistry 23, 3603-3609

9. LAEMMLI U. K. (1970) Cleavage of structural proteins during the assembly of the head of bacteriophage $\mathrm{T}_{4}$. Nature 227, $680-685$

10. Misono K. S. and Inagami T. (1980) Characterization of the active site of mouse submaxillary gland renin. Biochemistry 19, 2615-2622

11. Murakami K., Takahashi S., Suzuki F., Hirose S. and INAGAMI T. (1980) Intermediate molecular weight renin and renin-binding protein(s) in the hog kidney. Biomedical Res. 1, 392-399

12. Nagase M., Suzuki F., Fukamizu A., Takeda N., Takeuchi K., Murakami K. and NaKamura Y. (1994) Sequencing and expression of sheep angiotensinogen cDNA. Biosci. Biotechnol. Biochem. 58, 1884-1885

13. Nagase M., Suzuki F., Takahashi A., Fujimori M., SaWaI Y. and NaKamura Y. (1995) pH-dependent inactivation and reactivation of recombinant sheep angiotensinogen. Biosci. Biotechnol. Biochem. 59, 765-766

14. Nakamura N., Satomura S., Matsumura S. and MURAKAMI K. (1991) Identification of the active site of human renin with use of a new fluorogenic peptide. $J$. Biochem. 109, 741-745

15. Ohkubo H., Kageyama R., Ujihara M., Inayama S. and NaKanishi S. (1983) Cloning and sequence analysis of cDNA for rat angiotensinogen. Proc. Natl. Acad. Sci. USA 80, 2196-2200

16. OUCHTERLONY Ö. (1948) Antigen-antibody reactions in gels. Arkiv Kemi Mineral. Geol. 26, 1-9

17. PAGe I. H. and Helmer O. M. (1940) A crystalline pressor substance (angiotonin) resulting from the reaction between Mulhead renin activator. J. Exp. Med. 71, 29-42

18. Poorman R. A., Palermo D. P., Post L. E., Murakami K. Kinner J. H., Smith C. W., Reardon I. and Heinrikson R. L. (1986) Isolation and characterization of native human renin derived from Chinese hamster ovary cells. ProteinStruct. Funct. Genet. 1, 139-145

19. Printz M. P., Printz J. M. and Dworschack R. T. (1977) Human angiotensinogen. J. Biol. Chem. 252, 1654-1662

20. Skinner S. L., Dunn J. R., Mazzetti J., Campbell P. J. and FIDGE N. H. (1975) Purification, properties and kinetics of sheep and human renin substrates. Aust. J. Exp. Biol. Med. Sci. 53, 77-88

21. Suzuki F., Yamashita S., Takahashi A., Ito M., MiyaZAKI S., NAGATA Y. and NaKamURA Y. (1990) Highly sensitive microplate-ELISA for angiotensin I using $3,3^{\prime}, 5,5^{\prime}-$ tetramethylbenzidine. Clin. Exp. Hypertens. 12, 83-95

22. Tewksbury D. A., Dart R. A. and Travis J. (1981) The amino terminal amino acid sequence of human angiotensinogen. Biochem. Biophys. Res. Conmun. 99, 1311-1315

23. Yamauchi T., Suzuki F., Takahashi A., Tsutsumi I., Hori H., Watanabe T., Ishizuka Y., Nakamura Y. and Murakami K. (1992) Expression of rat renin in mammalian cells and its purification. Clin. Exp. Hypertens. 14, 377-392 